\title{
Autoantibodies as reporters identifying aberrant cellular mechanisms in tumorigenesis
}

\author{
Eng M. Tan \\ The Scripps Research Institute, MEM131, 10550 N. Torrey Pines Road, La Jolla, California 92037, USA. \\ Phone: (858) 784-8686; Fax: (858) 784-2131; E-mail: emtan@scripps.edu.
}

J. Clin. Invest. 108:1411-1415 (2001). DOI:10.1172/JCI200114451.

A common, if not intrinsic, feature of autoimmunity is a humoral immune response manifested by the production of autoantibodies targeted against self-cellular proteins and nucleic acids (1). Although first studied comprehensively in rheumatic autoimmune disease, autoantibodies and autoimmunity have now become major areas of research in many other illnesses, including type 1 diabetes and other diseases discussed in this Perspective series (2-4); the paraneoplastic neurological disorder (PND) syndromes $(5,6)$; bullous skin diseases; liver disease; and many others (for review see ref. 7). Among the many known autoantigens are several ubiquitous cellular proteins involved in fundamental cellular functions such as DNA replication and transcription. The process by which these and other self-molecules become immunogenic is not yet understood (8).

The study of tumor-associated autoimmunity may offer insights not only into the pathogenesis of autoimmune diseases in general, but perhaps also into the events that drive certain classes of cancer. Thus, changes in the structure or expression of certain self proteins, occurring during tumorigenesis, suggest mechanisms by which the immune system could be primed to perceive tumor-associated epitopes as foreign. One major route to cancer autoimmunity involves genetic mutations resulting in the production of defective p53 tumor suppressor protein. Another pathway, active in individuals with liver cancer, involves the aberrant expression of specific mRNA-binding proteins that may induce cell proliferation by preventing the turnover of mRNAs for growth factors. Other classes of mRNA-binding protein are normally restricted to the immunologically privileged domain of the brain but can become expressed ectopically and represent autoantigens in the PND syndromes. Together, these findings highlight the importance of altered post-transcriptional control at different stages of tumorigenesis.

\section{Autoantibodies in cancer patients with $p 53$ mutations}

The important role of $\mathrm{p} 53$ in tumorigenesis was reported from several laboratories in $1979(9,10)$, and thereafter, mutations in $p 53$ were found to be associated with most types of human cancers (11). p53 is a tumor suppressor factor, and its main function lies in its ability to transactivate a number of genes involved in cell cycle regulation leading to arrest of cell cycle progression and the repair of damaged or mutated DNA (12, 13). Antibodies to p53 in the sera of cancer patients were reported in 1982 (14), and in many subsequent studies. The reported frequency of antibodies in cancer has varied widely, ranging from $2.7 \%$ in prostate cancer to $31 \%$ in esophageal cancer, in part due to variability of the assay systems used (15). Although the sensitivity of p53 antibodies in diagnosis is not high, these antibodies are so rare in healthy people that the test is $98-99 \%$ specific $(15,16)$, making anti-p53 antibodies a dependable marker for cancer.

An important study correlating $p 53$ mutations with antibodies in lung cancer appeared in 1992 (17). Cancer cell lines from patients were analyzed for $p 53$ point mutations, stop codon mutations, and frameshift mutations. Patients with point mutations had single amino acid substitutions, which resulted in the accumulation of proteins with altered functions and increased stability relative to the rapidly degraded wildtype p53. These missense mutations occurred in the hot spot regions of $p 53$ in exons 4 to 9 . This study showed a good correlation between increased cellular accumulation of abnormal p53 and the presence of anti-p53 antibodies.

Contrary to what might have been expected, analysis of p53 antibody specificities revealed that the antigenic determinants on p53 resided not in the mutated hot spot regions but at the amino- and carboxy-terminal regions away from the central region of $\mathrm{p} 53$ containing the mutations $(18,19)$. The absence of antibodies in patients with stop codon mutations or frameshift mutations was largely related to the absence of p53 protein in the transformed cells (17). This appears to be a reasonable explanation for the known observation that although about $50 \%$ of human cancers have $p 53$ gene mutations (11), slightly less than half of these have circulating antibody (15). Thus, the antigenic load of accumulated aberrant protein appeared to be an important trigger of the autoantibody response. Another factor to consider is the distribution of p53, which is normally 
localized in the nucleus; in cancer, this protein can be excluded from the nucleus or localized in both nucleus and cytoplasm (20). Whether mutations with overexpression of abnormal p53 and aberrant localization are factors that are sufficient by themselves to stimulate autoimmune response remains to be clarified.

The observation that autoantibodies are equally reactive with wild-type and mutated p53 reflects the situation in many other autoimmune diseases, where antibodies are reactive with native cellular proteins (21). Thus normal p53 is expressed transiently and rapidly degraded, but mutant forms that are more resistant to degradation can accumulate in the nucleus and, aberrantly, in the cytoplasm. The challenging questions are whether such features are triggers that are sufficient to break tolerance in the immune system and how generalized these processes might be in other illnesses with autoimmune reactions.

Although a few studies have reported better prognosis in individuals with p53 antibodies, most work with breast, gastric, colon, and lung cancer patients associates these antibodies with bad prognosis and poorly differentiated tumors (15). This is not surprising if one regards the antibody response to p53 as a reaction to the antigenic load of abnormal or dysregulated $\mathrm{p} 53$. In addition, the clinical outcome might be significantly affected by the magnitude of cell-mediated immunity, which is known to be actively involved in tumor immune responses (22). Unfortunately, the cellular arm of the immune response has not been analyzed in most reports on clinical outcomes associated with p53 and other tumor-associated antibodies.

Another important clinical question is whether antip53 antibodies might be used as early markers of incipient tumors in populations at high risk. In 1993, we reported that one-third of patients with chronic hepatitis and/or liver cirrhosis suddenly showed increasing antibody titers and novel autoantibodies at or before transition to hepatocellular carcinoma (HCC) (23). p53 antibodies have also been examined retrospectively in heavy smokers and in patients with chronic obstructive pulmonary disease; anti-p53 antibodies have in some cases been detected several months before clinical diagnosis $(24,25)$. Thus, during incipient tumorigenesis, the immune system may sometimes sense abnormalities of cellular proteins involved in transformation before clinical symptoms appear or traditional diagnostic procedures detect the tumor. However, not all

\section{Table 1}

Changes in autoantibody responses associated with transformation to $\mathrm{HCC}$ in chronic liver disease patients

Category of change

Seroconversion from ANA negative to positive

Increase in ANA titer in previously positive ANA

Appearance of neoantibody (WB)

in previously positive ANA

All categories

ANA, antinuclear antibody by immunohistochemistry; WB, Western immunoblot. autoimmune reactions may be directed against tumorassociated antigens. The importance of differentiating between tumor-associated and non-tumor-associated responses is discussed below.

\section{Autoantibodies and the dysregulation}

of mRNA-binding proteins

HCC is unusual in that it is possible to identify individuals, such as those with liver cirrhosis and chronic hepatitis, who are at high risk of developing the disease over a period of several years (26). During conversion from chronic liver disease to HCC, the percentage of autoantibody-positive individuals rises significantly. In one study (23), one-third of HCC patients for whom serial serum samples were available before and at the time of diagnosis showed changes in autoantibody status (Table 1). The changes took place in the background of an already elevated frequency of autoantibodies in patients with chronic liver disease, suggesting that additional immunogenic stimuli were associated with tumorigenesis. Most patients manifested neoantibody responses, while the others showed increases in titers of pre-existing autoantibodies.

Sera showing the presence of neoantibodies have been used to immunoscreen cDNA expression libraries in order to isolate the putative tumor-associated antigens, a technique that was earlier used to isolate autoimmune rheumatic disease antigens. Proteins isolated with such tumor-associated antibodies include a serine-arginine repeat-bearing protein that acts in alternative mRNA splicing (27) and a cell cycle-related protein that represents a subunit of protein phosphatase 2A (28-30). Although this technique has been abundantly successful in isolating and characterizing many tumor-associated antigens, including those associated with PND syndromes $(5,6)$ and cancer/testis antigens (31), it remains possible that some of these molecules were antigenic even before the development of the tumor. This distinction can be made directly when premalignancy sera are available for a given patient, although tumor specificity of the antigens can also be established by showing that antibodies are present predominantly in patients with cancer.

In early studies on HCC, the tumor-associated autoantigens identified appeared to be idiosyncratic features of individual patients $(27,28)$, but more recent work has identified antigen-antibody systems that occur at relatively high frequency among cancer patients. Thus, antibodies to the nuclear protein cyclin $\mathrm{B} 1$, which is expressed in the $\mathrm{S}$ and $\mathrm{G} 2$ phases of the cell cycle, have been found in $15 \%(15 / 100)$ of patients with HCC, but in the same cohort no antibodies were detected to cyclins D1 and E and only one patient had antibodies to cyclin A (32). This apparent dominance of autoimmunity to cyclin B1 among many cell cycle-regulating proteins is as yet unexplained, but the finding of interest here is the presence of autoantibody to a single self protein in a significant subset of patients.

Analysis of sera from a large cohort of Chinese HCC patients identified a common 62-kDa autoantigen, p62, which has since been cloned and identified. This protein 
Table 2

Messenger RNA-binding proteins with RNP consensus sequence (RNA recognition motifs) and KH (hnRNP-K homology) domains involved in tumorigenesis

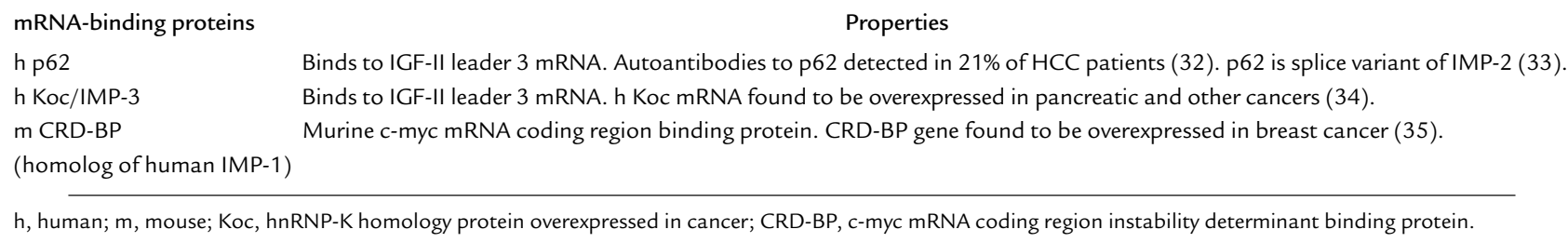

h, human; m, mouse; Koc, hnRNP-K homology protein overexpressed in cancer; CRD-BP, c-myc mRNA coding region instability determinant binding protein

\section{Properties}

Binds to IGF-II leader 3 mRNA. Autoantibodies to p62 detected in 21\% of HCC patients (32). p62 is splice variant of IMP-2 (33). Binds to IGF-II leader 3 mRNA. h Koc mRNA found to be overexpressed in pancreatic and other cancers (34). Murine c-myc mRNA coding region binding protein. CRD-BP gene found to be overexpressed in breast cancer (35).

carries two types of RNA-binding motifs, an RNP consensus sequence motif in the amino-terminal region and four repeats of an hnRNP-K homology $(\mathrm{KH})$ motif in the C-terminal two-thirds of the protein (33). Twenty-one percent of this group of HCC patients had antibodies to p62, whereas patients with liver cirrhosis, patients with chronic hepatitis, and normal controls were negative, indicating that the autoimmune response was highly HCC-related. Several recent studies on related proteins have now added to the potential significance of these observations. Nielsen et al. (34), studying cellular proteins that bind to IGF-II mRNAs in a rhabdomyosarcoma cell line, isolated three proteins that bind the $5^{\prime}$ untranslated region found in one IGF-II mRNA species (leader 3 IGF-II mRNA), which is developmentally regulated and expressed in fetal tissue but not adult tissue. These IGF-II mRNA binding proteins, called IMP-1, -2, and -3 , all contained the RNP consensus sequence and KH RNA-binding motifs. p62 and IMP-2 are alternatively spliced products of the same gene.

The relationship of this family of mRNA-binding proteins to cancer has been shown in two studies besides our own (Table 2). Mueller-Pillasch et al. (35) isolated a cDNA overexpressed in human pancreatic cancer by differential screening of cancer versus normal tissues. The deduced protein called Koc (KH protein overexpressed in cancer), which is identical to IMP-3, is overexpressed in several other types of cancer. Doyle et al., who had earlier isolated c-myc mRNA coding region instability determinant binding protein (CRD-BP), a murine homolog of human IMP-1 (Table 2) that binds a 250-nucleotide sequence in the coding region of c-myc mRNA, have recently shown amplification of the gene encoding this protein in human breast cancer (36). Previously, these investigators had shown in cell-free mRNA decay experiments that CRD-BP acts as a shield to protect c-myc mRNA from rapid degradation by binding to the 250 nucleotide instability determinant (37).

The studies showing that p62 and Koc are mRNAbinding proteins and that one of the targets is IGF-II mRNA have interesting implications with regard to cancer. IGF-II is a growth factor whose overproduction has been shown to promote tumorigenesis (38), and its mRNA is specifically overexpressed in HCC tumor nodules (39). IGF-II transgenic mice have increased frequency of diverse malignancies (40), and in SV40 oncogene-induced tumorigenesis, IGF-II serves as an accessory factor in transformation (41). Similarly, as Pasquinelli et al. (42) have observed, transgenic mice expressing the hepatitis B virus envelope exhibit chronic hepatocellular injury and inflammation, leading to successive cycles of regenerative hyperplasia and inflammation and ultimately to development of HCC. These authors found no abnormalities in the structure or expression of a large panel of oncogenes and tumor suppressors, including ras, myc, fos, abl, src, Rb, and p53, but noted that IGF-II was consistently overexpressed.

These data suggest that IGF-II induction is common to HCC tumors induced by a variety of stimuli and raise the question of whether the IGF-II mRNA dysregulation is a necessary step in this disease pathway. Indeed, we have observed by immunohistochemistry that in one-third (9/27) of patients with HCC, cancer nodules showed abundant immunostaining for p62 protein whereas adjacent nonmalignant hepatic parenchymal and other cells were devoid of detectable p62 (Figure 1a). In one of two cases of cholangiocarcinoma, malignant bile duct epithelial cells also expressed p62 (Figure 1b). Nine specimens of normal human adult liver did not contain detectable p62, whereas fetal liver contained immunoreactive p 62 in hepatic parenchymal cells (43). Hence, p62 is a developmentally regulated cellular protein expressed in fetal liver but not in adult liver and aberrantly expressed in HCC. We propose that, analogous to the manner in which CRD-BP protects c-myc mRNA from degradation, p62 and Koc might allow expression of fetal
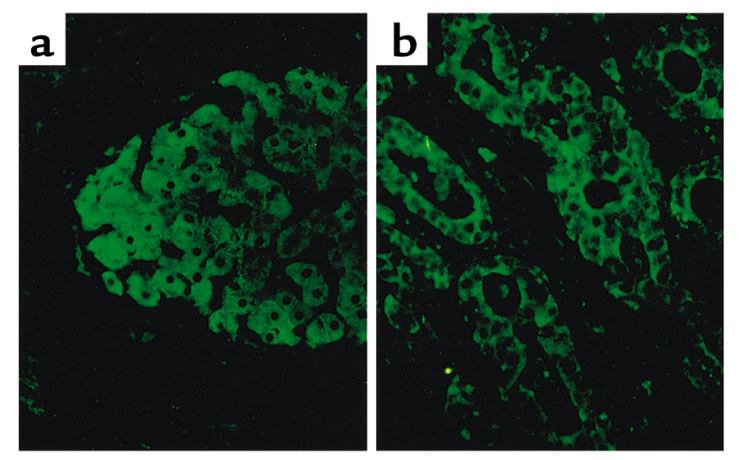

\section{Figure 1}

Tumor nodule from a patient with HCC examined by immunohistochemistry (a), showing expression of $\mathrm{p} 62$ protein in cytoplasm of cancer cells but no detectable p62 in hepatic or other cells in adjacent noncancer areas. One of two cholangiocarcinoma patients showed expression of p62 in cytoplasm of malignant bile duct cells (b). 
IGF-II mRNA in malignant cells, resulting in overproduction of IGF-II growth factor.

\section{Aberrantly expressed neuronal proteins as autoantigens in PND}

Like p62 and Koc, Hu antigen-containing proteins represent RNA-binding autoantigens whose participation in tumorigenesis was first suspected because of the identification of autoantibodies in cancer patients. A number of neurological disorders, such as sensory neuronopathy, opsoclonus myoclonus ataxia, cerebellar degeneration, and limbic and brain stem encephalitis, are overt clinical symptoms in patients with tumors of the lung, breast, ovary, and testis and have been collectively called PND syndromes $(5,6)$. PND patients make antibodies to proteins that are normally neuron-specific but that become expressed in non-neuronal tumors.

Two classes of these proteins have been extensively analyzed and have been shown to be RNA-binding proteins. The $\mathrm{Hu}$ proteins are antigens in paraneoplastic sensory neuronopathy, are highly homologous to the Drosophila protein ELAV (embryonic lethal abnormal vision), and contain three RNA recognition motifs. The ELAV/Hu proteins appear to have the function of binding to the f-rich element in the $3^{\prime}$ untranslated regions of many protooncogene and cytokine messenger RNAs, such as c-myc, c-fos, c-myb, and GM-CSF. These AUrich regions are instability elements, and removal of these elements results in the stabilization of transcripts such as GM-CSF mRNA. Placing the AU-rich element from GM-CSF into the $3^{\prime}$ UTR of a stable mRNA destabilizes the latter. Studies by Keene and others show that the ELAV/Hu proteins promote the stability of the AUrich mRNAs, leading to greater production of translation products (44).

A second group of autoantigens in the PND syndromes are proteins of the Nova group, which are KHrelated RNA-binding proteins (6). Other autoantigens in PND syndromes continue to be described (45). Although binding of proteins such as $\mathrm{Hu}$ and Nova to RNA has been clearly established, the effect of this binding on target RNA function has not been definitively established. Much as p53 abnormalities are associated with antibody responses in only a subset of patients, it appears that all small-cell lung tumors express the Hu antigen but a smaller percentage produce autoantibodies (46).

\section{Future directions}

The humoral immune responses to tumor-associated autoantigens show clearly that antibodies can be useful reagents to identify aberrant cellular mechanisms involved in tumorigenesis. Just as many rheumatic autoantibodies have been important reagents to help in identifying molecules involved in pre-mRNA splicing, cell division, and DNA replication (1), cancer autoantibodies might be anticipated to provide some insights into pathways associated with cell transformation, particularly the post-transcriptional regulation of growth factors and cytokines. Many tumorassociated autoantigens have already been identified
(47). As more are found, it will be important, but not always straightforward (48), to determine which ones are directly related to tumorigenesis.

Success in the treatment of cancer is influenced a great deal by early detection. Clearly, the immune system is capable of sensing at least some tumor-associated dysregulated cell functions before many standard clinical tests for cancer detection. Might certain autoantibodies therefore be added to the array of diagnostic tests for cancer - or even for early detection, as now appears to be possible in tests for the characteristic antibodies that presage type 1 diabetes (2)? Although genetic abnormalities of p53 have been shown to be present in up to $50 \%$ of all cancers (11), the occurrence of autoantibodies is in the range of 15-20 percent (15). We have recently observed that some cancer patients produce antibodies to more than one tumor-associated antigen (49). In a study using a multiple tumor-associated antigen array of six antigens, it was found that in 546 cancer sera examined, $45 \%$ of the sera were positive for antibody to any one of the six antigens, compared with $12 \%$ when a single antigen was used (our unpublished results). With the expansion of the multiple antigen array to include other tumor-associated autoantigens, the sensitivity of this detection system could be further increased.

Other uses of the knowledge brought by autoantibodies regarding cellular molecules involved in tumorigenesis include cancer therapy aimed at restoring normal function or at abrogating harmful effects caused by dysregulation of certain cellular functions. Efforts in these directions are underway, including development of cancer vaccines with p53 as the target (50). Other major tumor-associated autoantigens could be reasonable targets for cancer therapy. In clinical trials associated with the development of such therapy, it may prove efficient to select subjects with detectable autoantibodies, using the immune response as an indication of which patients have the abnormal cellular mechanisms being targeted for treatment.

\section{Acknowledgments}

This work was supported by a grant from the National Cancer Institute. This is publication no. 13898-MEM from The Scripps Research Institute. The contribution of colleagues in my laboratory, J.-Y. Zhang, M. Lu, W. Zhu, and E.K.L. Chan, in many aspects of this work is gratefully acknowledged.

1. Tan, E.M. 1989. Antinuclear antibodies: diagnostic markers for autoimmune diseases and probes for cell biology. Adv. Immunol. 44:93-151.

2. Notkins, A., and Lernmark, A. 2001. Autoimmune type 1 diabetes: resolved and unresolved issues. J. Clin. Invest. 108:1247-1252.

3. Rapoport, B., and McLachlan, S.M. 2001. Thyroid autoimmunity. $J$. Clin. Invest. 108:1253-1259.

4. Papadopoulos, G.K., Wijmenga, C., and Koning, F. 2001. Interplay between genetics and the environment in the development of celiac disease: perspectives for a healthy life. J. Clin. Invest. 108:1261-1266.

5. Szabo, A., et al. 1991. HuD, a paraneoplastic encephalomyelitis antigen, contains RNA-binding domains and is homologous to Elav and sex-lethal. Cell. 67:325-333.

6. Darnell, R.B. 1996. 1996. Onconeural antigens and the paraneoplastic disorders: at the intersection of cancer, immunity and the brain. Proc. Natl. Acad. Sci. USA. 93:4529-4536. 
7. Rose, N.R., and Mackay, I.R. 1998. The autoimmune diseases. Academic Press. New York, New York, USA.

8. Zinkernagel, R. 2000. What is missing in immunology to understand immunity? Nat. Immunol. 1:181-185.

9. Linzer, D.I.H., and Levine, A.J. 1979. Characterization of a $54 \mathrm{~K}$ dalton cellular SV40 tumor antigen present in SV40-transformed cells and uninfected embryonal carcinoma cells. Cell. 17:43-52.

10. DeLeo, A.B., et al. 1979. Detection of a transformation-related antigen in chemically induced sarcomas and other transformed cells of the mouse. Proc. Natl. Acad. Sci. USA. 76:2420-2424.

11. Hollstein, M.B., Sidransky, D., Vogelstein, B., and Harris, C.C. 1991. p53 mutations in human cancer. Science. 253:49-53.

12. Kastan, M.B., Onyekwere, O., Sidransky, D., Vogelstein, B., and Craig, R.W. 1991. Participation of p53 protein in the cellular response to DNA damage. Cancer Res. 51:6304-6311.

13. Vogelstein, B., Lane, D., and Levine, A.J. 2000. Surfing the p53 net. Nature. 408:307-310.

14. Crawford, L.V., Pim, D.C., and Bulbrook, R.D. 1982. Detection of antibodies against the cellular protein p53 in sera from patients with breast cancer. Int. J. Cancer. 30:403-408.

15. Soussi, T. 2000. Antibodies in the sera of patients with various types of cancer: a review. Cancer Res. 60:1777-1788.

16. Vogl, F.D., Frey, M., Kreienberg, R., and Runnebaum, I.B. 2000. Autoimmunity against $\mathrm{p} 53$ predicts invasive cancer with poor survival in patients with an ovarian mass. Br. J. Cancer. 83:1338-1343.

17. Winter, S.J., et al. 1992. Development of antibodies against p53 in lung cancer patients appears to be dependent on the type of p53 mutations. Cancer Res. 52:4168-4174.

18. Lubin, R., Schlichtholz, B., Bengoufa, D., and Soussi, T. 1993. Analysis of $\mathrm{p} 53$ antibodies in patients with various cancers define B-cell epitopes of human p53: distribution on primary structure and exposure on protein surface. Cancer Res. 53:5872-5876.

19. Labrecque, S., Naftaly, N., Thomson, D., and Matlaskewski, G. 1993. Analysis of the anti-p53 antibody response in cancer patients. Cancer Res. 53:3468-3471.

20. Moll, U.M., Riou G., and Levine, A.J. 1992. Two distinct mechanisms alter p53 in breast cancer: mutation and nuclear exclusion. Proc. Natl. Acad. Sci. USA. 89:7262-7266.

21. Tan, E.M., Chan, E.K.L., Sullivan K.F., and Rubin, R.L. 1988. Antinuclear antibodies (ANAs): diagnostically specific immune markers and clues towards the understanding of systemic autoimmunity. Clin. Immunol. 47:121-141.

22. Boon, T., and Van der Bruggen, P. 1996. Human tumor antigens recognized by T lymphocytes. J. Exp. Med. 183:725-729.

23. Imai, H., Nakano, Y., Kiyosawa, K., and Tan, E.M. 1993. Increasing titers and changing specificities of antinuclear antibodies in patients with chronic liver disease who develop hepatocellular carcinoma. Can cer. 71:26-35.

24. Lubin, R., et al. 1995. Serum p53 antibodies as early markers of lung cancer. Nat. Med. 1:701-702.

25. Trivers, G.E., et al. 1996. Anti-p53 antibodies in sera from patients with chronic obstructive pulmonary disease can predate a diagnosis of cancer. Cancer Res. 2:1767-1775.

26. Popper, H., Schafritz, D.A., and Hoofnagle, J.H. 1987. Relation of the hepatitis B virus carrier state to hepatocellular carcinoma. Hepatology. 7:764-772.

27. Imai, H., Chan, E.K.L., Kiyosawa, K., Fu, X.-D., and Tan, E.M. 1993. Novel nuclear autoantigen with splicing factor motifs identified with antibody from hepatocellular carcinoma. J. Clin. Invest. 92:2419-2426.

28. Muro, Y., Chan, E.K.L., Landberg, G., and Tan, E.M. 1995. A cell-cycle nuclear autoantigen containing WD-40 motifs expressed mainly in S and G2 phase cells. Biochem. Biophys. Res. Commun. 207:1029-1037.

29. Moreno, C.S., et al. 2000. WD40 repeat proteins striatin and SG2 nuclear autoantigen are members of a novel family of calmodulin- binding proteins that associate with protein phosphatase 2A. J. Biol. Chem. 275:5257-5263.

30. Zhu, W., Chan, E.K.L., Li, J., Hemmerich, P., and Tan, E.M. 2001. Transcription activating property of autoantigen SG2NA and modulating effect of WD-40 repeats. Exp. Cell Res. 269:312-321.

31. Stockert, E., et al. 1998. A survey of the humoral immune response of cancer patients to a panel of human tumor antigens. J. Exp. Med. 187:1349-1354.

32. Covini, G., et al. 1997. Immune response to cyclin B1 in hepatocellular carcinoma. Hepatology. 25:75-80.

33. Zhang, J.-Y., Chan, E.K.L., Peng, X.-X., and Tan, E.M. 1999. A novel cytoplasmic protein with RNA-binding motifs is an autoantigen in human hepatocellular carcinoma. J. Exp. Med. 189:1101-1110.

34. Nielsen, J., et al. 1999. A family of insulin-like growth factor II mRNA binding proteins represses translation in late development. Mol. Cell. Biol. 19:1262-1270.

35. Mueller-Pillasch, F., et al. 1997. Cloning of a gene highly expressed in cancer coding for a novel $\mathrm{KH}$-domain containing protein. Oncogene. 14:2729-2733.

36. Doyle, G.A., Bourdeaux-Heller, J.M., Coulthard, S., Meisner, L.F., and Ross, J. 2000. Amplification in human breast cancer of a gene encoding a c-myc mRNA binding protein. Cancer Res. 60:2756-2759.

37. Doyle, G.A., et al. 1998. The c-myc coding region determinant binding protein; a member of a family of $\mathrm{KH}$ domain RNA binding proteins. Nucleic Acids Res. 26:5036-5044.

38. Westley, B.R., and May, F.E.B. 1995. Insulin-like growth factors: the unrecognised oncogenes. Br. J. Cancer. 72:1065-1066.

39. Cariani, E., et al. 1988. Differential expression of insulin-like growth factor II mRNA in human primary liver cancers, benign liver tumors and liver cirrhosis. Cancer Res. 48:6844-6849.

40. Rogler, C.E., et al. 1994. Altered body composition and increased frequency of diverse malignancies in insulin-like growth factor II transgenic mice. J. Biol. Chem. 269:13779-13784.

41. Christofori, G., Naik, P., and Hanahan, D. 1994. A second signal supplied by insulin-like growth factor II in oncogene-induced tumorigenesis. Nature. 369:414-417.

42. Pasquinelli, C., Bhavani, K., and Chisari, F. 1992. Multiple oncogenes and tumor suppressor genes are structurally and functionally intact during hepatocarcinogenesis in hepatitis B transgenic mice. Cancer Res. 52:2823-2829.

43. Lu, M., et al. 2001. Aberrant expression of fetal RNA-binding protein p62 in liver cancer and liver cirrhosis. Am. J. Pathol. 159:945-953.

44. Keene, J.D. 1999. Why is Hu where? Shuttling of early response gene messenger RNA subsets. Proc. Natl. Acad. Sci. USA. 96:5-7.

45. Voltz, R., Gultekin, H., and Rosenfeld, M.R. 1999. A serological marker of paraneoplastic limbic and brain stem encephalitis in patients with testicular cancer. N. Engl. J. Med. 340:1788-1795.

46. Dalmau, J., Furneaux, H.M., Cordon-Cardo, C., and Posner, J.B. 1992. The expression of the $\mathrm{Hu}$ (paraneoplastic encephalomyelitis/sensory neuronopathy) antigen in human normal and tumor tissues. Am. J. Pathol. 141:881-886.

47. Conrad, K. 2000. Autoantibodies in cancer patients and in persons with a higher risk of cancer development. In Cancer and autoimmunity. Y. Shoenfeld and M.E. Gershwin, editors. Elsevier Science. Amsterdam, The Netherlands. 159-173.

48. Hanahan, D., and Weinberg, R.A. 2000. The hallmarks of cancer. Cell. 100:57-70.

49. Zhang, J.-Y., Zhu, W., Imai, H., Kiyosawa, K., Chan, E.K.L. and Tan, E.M. 2001. De novo humoral immune response to cancer-associated autoantigens during transition from chronic liver disease to hepatocellular carcinoma. Clin. Exp. Immunol. 125:3-9.

50. Roth, J., et al. 1996. p53 as a target for cancer vaccines: recombinant canarypox virus vectors expressing p53 protect mice against lethal tumor challenge. Proc. Natl. Acad. Sci. USA. 93:4781-4786. 